DOI: http://dx.doi.org//10.1590/1981-7746-sol00235

\title{
ESTRESSE OCUPACIONAL NA MÍDIA IMPRESSA: UMA PERSPECTIVA DE CHRISTOPHE DEJOURS
}

\author{
OCCUPATIONAL STRESS IN THE PRINTED MEDIA: \\ A CHRISTOPHE DEJOURS PERSPECTIVE
}

\author{
Átala Lotti Garcia ${ }^{1,2}$ (iD [0000-0002-1563-4837], Tatiana Breder Emerich² (iD [0000-0003-4104-0562], \\ Luciane Bresciani Salaroli3 ${ }^{\text {(iD }}$ [0000-0002-1881-0306], Aline Guio Cavaca ${ }^{2}$ iD [0000-0001-7314-584x], Edson \\ Theodoro dos Santos Neto² (iD) [0000-0002-7351-7719]
}

1 Universidade Federal de Minas Gerais, Programa de Educação, Belo Horizonte, MG, Brasil. <algacia.lotti@gmail.com>

2 Universidade Federal do Espírito Santo, Programa de Pós-graduação em Saúde Coletiva, Vitória, Espírito Santo, Brasil.

${ }^{3}$ Universidade Federal do Espírito Santo, Programa de Pós-graduação em Saúde Coletiva e Programa de Pós-graduação em Nutrição e Saúde, Vitória, Espírito Santo, Brasil.

Resumo O estresse no trabalho afeta os aspectos psicológicos, físicos e sociais do trabalhador, bem como a eficácia das organizações produtivas. O objetivo deste estudo foi compreender o estresse ocupacional na mídia jornalística sob a ótica da organização do trabalho. Trata-se de uma pesquisa qualitativa conduzida por análise de conteúdo de Bardin, com base nas categorias analíticas previamente definidas pela Organização Mundial da Saúde, no relatório "Work Organization \& Stress", sob a perspectiva do referencial teórico de Christophe Dejours. Foram classificadas 727 notícias que tinham como palavra-chave 'estresse', sendo 130 notícias específicas do tema estresse no trabalho. Dentre estas notícias, foram classificadas as categorias "Conteúdo dos Riscos do Estresse" (47) e "Contexto dos Riscos do Estresse" (59), ambas com suas subcategorias. Constatou-se que a mídia quase não tem funcionado como porta-voz dos trabalhadores a fim de cumprir seu papel social, sem estimulá-los como sujeitos sociais. Propõe-se que a mídia jornalística estimule ações que impulsionem o protagonismo dos trabalhadores, baseado na visibilidade das legislações vigentes, e aponte caminhos mais diretos para que os trabalhadores possam lutar pela garantia de direitos à saúde no combate ao estresse, com perspectiva crítica do entendimento sobre organização do trabalho e suas potencialidades na promoção da saúde.

Palavras-chave meios de comunicação; estresse ocupacional; jornada de trabalho.
Abstract Stress at work affects psychological, physical and social aspects of the workers, as well as the efficacy of the productive organizations. The goal of the present study was to comprehend occupational stress in the journalistic media under the perspective of the organization of labor. It is a qualitative research conducted through Bardin's content analysis, based on the analytical categories previously defined by the World Health Organization in the 'Work Organization \& Stress' report, under the perspective of the theoretical framework of Christophe Dejours. We classified 727 news stories that had'stress' as a keyword, with 130 news stories specifically approaching the topic of stress at work. The news stories were divided into two categories, each with subcategories of their own:'Content of the Risks of Stress' (47) and 'Context of the Risks of Stress' (59). We verified that the media has scarcely been working as the spokesperson for the workers in order to fulfill its social role, without stimulating them as social subjects. We propose that the news media stimulates actions that boost the prominence of the workers, based on the visibility of the current laws, and indicates more direct ways for the workers to be able to fight to guarantee their rights to health in the struggle against stress, with a critical perspective of the understanding regarding the organization of labor and its potentialities in the promotion of health. Keywords communications media; occupational stress; work hours. 


\section{Introdução}

O estresse no trabalho afeta os aspectos psicológicos, físicos e sociais do trabalhador, bem como a eficácia das organizações (Leka, Griffiths e Cox, 2004; Kröll, Doebler e Nüesch, 2017). O estresse é o estado fisiológico e psicológico causado por estímulos externos e internos, tanto positivos quanto negativos (Selye, 1936, 1956; Pereira e Zille, 2010). As relações causais do estresse ocupacional estão relacionadas não apenas ao trabalhador e suas qualidades pessoais, mas também a componentes externos a ele, que são um conjunto de imposições que normatizam a dinâmica imposta pelo ambiente organizacional, incluindo as relações com os pares, a desvalorização do papel social, a dignidade do trabaIhador e as perdas financeiras (Cohen, Gianaros e Manuck, 2016). Dentro dessa perspectiva, a experiência de estresse depende das características, habilidades e competências individuais do trabalhador para lidar com as demandas impostas no ambiente de trabalho (Coté, 2005; Hackney e Perrewé, 2018), com redução de seus impactos.

Dentre os impactos do estresse ocupacional na saúde, inclui-se o fato de que o mundo do trabalho e suas mudanças têm requerido demandas crescentes dos trabalhadores, tais como: contratos temporários, maior carga e pressão do trabalho (Leka, Griffiths e Cox, 2004; European Agency for Health and Safety at Work, 2009), mudanças no papel feminino no mundo do trabalho (Gartzia, Pizarro e Baniandres, 2018), e desequilíbrios entre a vida profissional e pessoal (Proost et al., 2010; Fan et al., 2015; Sugawara et al., 2017; Smoktunowicz e Cieślak, 2018). O estresse ocupacional tem sido responsável por cerca de $50 \%$ a $60 \%$ de faltas no trabalho, representando diminuição da qualidade de vida e menor desempenho nas organizações (European Agency for Health and Safety at Work, 2009). O estresse ocupacional na psicodinâmica do trabalho se revela como elemento patológico (Dejours, 2008; Zeytinoglu et al., 2017) e de manifestação sintomática de doenças sistêmicas (Mendes, 2008; Nogueira e Freitas, 2015) e de doenças relacionadas ao trabalho (Ramazzini, 2016).

Devido aos impactos na saúde, o estresse ocupacional tem sido alvo de pesquisas científicas (Mark, 2008) que incluem avaliações da sobrecarga de estresse em diferentes categorias profissionais, inclusive entre jornalistas nos meios de comunicação que escrevem sobre o tema (Ertel et al., 2005).

Nesse sentido, pode-se destacar também o papel que os próprios meios de comunicação exercem sobre essa temática, visto que a mídia possui grande influência na sociedade com a divulgação do noticiário sobre estresse ocupacional, tais notícias são consideradas aprofundadas, pertinentes, contextualizadas e coerentes - ou não - (Lewis, Williams e Franklin, 2008). $\mathrm{O}$ estresse no trabalho tem sido um assunto de bastante visibilidade nos 
meios de comunicação (Danna e Griffin, 1999), impactando no imaginário social sobre o tema, visto que a grande maioria da população usa a mídia como principal fonte de informação, o que inclui as informações sobre saúde (Castell et al., 2014), sendo o jornal impresso um meio de comunicação que sintetiza e reverbera a circulação de notícias de outros meios de comunicação, como: rádio, TV e mídias sociais. Dessa forma, a divulgação na mídia sobre riscos do estresse ocupacional poderia ajudar os trabalhadores a serem mais ativos para superar as experiências negativas no trabalho.

Entre os referenciais teóricos aplicados aos estudos sobre o impacto do estresse no ambiente de trabalho, uma das possíveis referências a serem adotadas é o modelo explicativo que busca demonstrar que o estresse ocupacional está relacionado à organização do trabalho e que inclui aspectos psicológicos associados às consequências do trabalho, sejam elas positivas, decorrentes do prazer; ou negativas, advindas do sofrimento (Dejours, 1992, 1993, Melo, Cavalcante e Facanha, 2019).

Nesse sentido, Christophe Dejours (1992, 1994a, 1994b) desenvolveu estudos sobre a organização do trabalho, apontando as experiências de prazer e satisfação que são fontes de realização e responsáveis pelo processo de identidade dos sujeitos. Entretanto, há um efeito negativo que o trabalho pode produzir, o desprazer, que pode acarretar efeitos patológicos, trazendo consigo uma significativa redução do bem-estar e da qualidade de vida do trabalhador, produzindo um efeito patogênico nocivo à saúde.

Diante dessas considerações, este estudo objetiva compreender o estresse ocupacional noticiado na mídia jornalística impressa sob a ótica da organização do trabalho, tendo como referencial teórico a perspectiva de Christophe Dejours.

\section{Métodos}

Trata-se de um estudo transversal de abordagem qualitativa, o qual teve como território de abrangência as notícias veiculadas em jornais do Estado do Espírito Santo. O período de estudo foi de 1 de janeiro de 2015 a 30 de abril de 2017, período este que possibilita o panorama de noticiabilidade sobre o assunto, de acordo com novas pesquisas e tecnologias em saúde, planos, políticas e investimentos na área.

O estudo foi desenvolvido no Observatório de Saúde na Mídia - Regional Espírito Santo (OSM-ES), o qual acompanha e analisa criticamente os modos pelos quais os meios de comunicação constroem discursivamente os sentidos sobre os temas da saúde (Cavaca AG, Emerich, Lerner, 2016; Silva et al., 2018) e têm avançado com pesquisas e protocolos desenvolvidos 
em estudos recentes (Cavaca, AG et al., 2016; Coqueiro et al., 2018; Cavaca, SD et al., 2018).

A coleta de dados foi realizada por meio da busca de notícias que continham o radical 'stress' e seus derivados em suas palavras-chave. Como ferramenta de coleta de dados foi empregado para a busca, o programa Adobe Acrobat Reader DC. Esse programa possui o localizador de palavras/ termos como uma de suas ferramentas e permite que sejam localizados os textos que exibem os termos de interesse. Após o levantamento das notícias no período indicado, o material foi tratado no software de análise de dados qualitativos Maxqda versão 2018, que faz a organização do material empírico e auxilia no processo de codificação e categorização, via computador, bem como na recuperação do texto codificado, permitindo visualizar a frequência do código e criar gráficos e diagramas, ajudando o pesquisador a analisar sem viés (Kus Saillard, 2011).

Foram incluídas no estudo as matérias publicadas nos jornais "A Tribuna" e "A Gazeta", que faziam referência ao estresse relacionado ao trabalho. Foram excluídas as matérias que citaram o estresse em contexto irrelevante ao estudo, como propagandas, promoção de eventos, palavras sinônimas, classificados, saúde animal, entre outros.

A análise dos dados ocorreu fundamentada na análise de conteúdo, conforme preconizada por Lawrence Bardin, 2015. Assim, a cobertura midiática sobre o estresse no trabalho ocorreu em três etapas principais. A primeira etapa, composta pela pré-análise dos dados, incluiu a seleção das notícias que continham a temática do estresse com base nas das palavras-chave, leitura flutuante dessas notícias e organização do material mediante seleção dos trechos mais relevantes das notícias. Nessa etapa foram extraídas 727 notícias que continham como palavra-chave 'estresse', sendo 130 notícias específicas do tema 'estresse no trabalho'; após a leitura na integra da notícia, foram escolhidos os trechos de notícias mais significativos.

A segunda etapa foi a exploração do material, na qual as notícias foram incluídas nas categorias adotadas a priori pelos pesquisadores, com base no documento da Organização Mundial da Saúde (OMS), intitulado "Work Organization \& Stress" (Leka, Griffiths e Cox, 2004). Na busca refinada, procuraram-se notícias que representassem as categorias e suas subdivisões propostas pela OMS; a primeira categoria foi 'Conteúdo dos Riscos do Estresse', com quatro subcategorias: 'tarefas do trabalho', 'carga e ritmo de trabalho', 'horas de trabalho', e 'participação e controle'. A segunda categoria 
foi o 'Contexto dos Riscos do Estresse', com cinco subcategorias: 'desenvolvimento de carreira, status e pagamento', 'função na organização', 'as relações interprofissionais,' 'a cultura organizacional','a interface trabalho-família'. Vale ressaltar que uma matéria pode ter uma abordagem plural da temática do estresse, entretanto, optou-se pela categorização das 130 notícias sobre trabalho e estresse, segundo a perspectiva predominante da temática.

A terceira etapa, composta pelo tratamento dos resultados, incluiu a discussão dos trechos das notícias selecionadas em cada categoria com os artigos científicos que embasaram a interpretação e inferências dos resultados evidenciados pelos dados, sob o referencial da organização do trabalho de Christophe Dejours (1992). A discussão implicou o debate dos temas: trabalho e estresse, baseado no questionamento de problemas e na contestação de visões comuns acerca dos temas (Turato, 2005, 2011).

O estudo foi aprovado pelo Comitê de Ética em Pesquisas da Universidade Federal do Espírito Santo por meio do parecer CAAE 58948516.5.0000.5060, em 1 de setembro de 2016, como consequência da parceria com o Observatório de Saúde na Mídia - Regional ES, o qual possui aprovação para pesquisas desse âmbito no CEP, em conformidade com a Resolução 466/2012.

\section{Resultados e discussão}

A primeira categoria 'Conteúdo dos riscos do estresse' apresentou em suas subcategorias, o seguinte número de notícias, de forma decrescente para cada categoria: "carga e ritmo de trabalho' - 18 notícias; 'horas de trabalho' - 12 notícias; 'participação e controle' 10 notícias; 'tarefas do trabalho' - 7 notícias. A segunda categoria 'Contexto dos riscos do estresse', teve como número de notícias em cada subcategoria: 'função na organização' - 22 notícias; 'a cultura organizacional" - 16 notícias; 'desenvolvimento de carreira, status e pagamento' - 14 notícias; 'as relações interprofissionais' - 6 notícias; e 'a interface trabalho-família' - 1 notícia. Dentre as demais notícias, 10 não se aplicavam a nenhuma das subcategorias e 14 se incluíam em mais de uma subcategoria, e, por isso, não foram classificadas dentro de uma subcategoria específica.

De acordo com Rodrigues $(1997,2016)$ existem dois campos de estudo distintos: o campo da saúde e o campo jornalístico. Esses campos possuem características distintas ao lidar com o tema da saúde. O primeiro lida com a saúde segundo uma perspectiva discursiva esotérica em que há um discurso proferido por especialistas, com base em alguns critérios próprios deste campo. O segundo com uma perspectiva exotérica, ou seja, uma visão leiga que produz discursos para um público universal, isto é, não especializado, premido pela contingência do tempo, em que são mínimas as distâncias 
temporais entre a produção e a divulgação jornalística, em todos os trechos das notícias citados, ao longo do estudo, precisamos refletir e ter essas informações em consideração.

As categorias são apresentadas com base em trechos das notícias - as quais foram identificadas pela data e pelo periódico -, analisadas conforme referencial teórico científico, apresentados a seguir.

\section{Conteúdo dos riscos do estresse: tarefas do trabalho, carga de trabalho e ritmo de trabalho, horas de trabalho, participação e controle}

O conceito básico da teoria de Dejours (1992) se refere ao papel da organização do trabalho não só como divisão de tarefas, mas a divisão dos homens com seus desejos e aspirações. Se o trabalhador não tem motivação com a tarefa principal, a carga, o ritmo, as horas, a participação e a falta de controle são entendidas como um fardo e sofrimento. Nesse sentido, as tarefas do trabalho que não promovem a motivação ao trabalhador podem ser consideradas desagradáveis, aversivas, monótonas, repetitivas e desestimulantes. Como ilustrado no trecho a seguir:

... a redução do horário de intervalo dos trabalhadores pode ser taxada como "uma loucura". "Existem atividades que são muito repetitivas e estressantes, e o horário de descanso é um fator de segurança" disse... (A Tribuna-17/07/2015)

A notícia apresenta para o leitor uma informação importante sobre os temas de segurança e motivação, corroborando com autores Bao et al. (2016) e Stajkovic e Luthans (2003) que consideram a segurança no trabalho relacionada às tarefas estimulantes, com tempo adequado para ser realizada, a notícia apresentada destaca o contrário, atividades repetitivas como fator de insegurança.

Em relação à carga e ritmo de trabalho, uma maior ou menor intensidade de trabalho, sob pressão de tempo, pode ser um fator de risco de estresse ocupacional relevante para as doenças que mais acometem a saúde do trabalhador (Leka, Griffiths e Cox, 2004). Ao se remeter às características específicas do trabalho, está se colocando em análise o conteúdo da tarefa desenvolvida, o ritmo de determinada tarefa pelo tempo requerido para sua realização, a quantidade de horas trabalhadas, incluindo horas-extras, a existência de desvio de função, ou seja, a realização de tarefas não previstas na função original, e a necessidade de possuir mais de um emprego. Estudos mostram que as profissões que tem papéis muito diversificados das 
funções e lidam com o público, como professores e profissionais de saúde, têm alto nível de estresse (Albuquerque et al., 2018; Aredes, Giacomin e Firmo, 2018). O estresse é um risco inerente a essas profissões, assim como a carga de trabalho, que pode gerar pânico e outros distúrbios, evidenciados nos trechos das notícias:

"Tive crise de pânico por causa do estresse devido à sobrecarga de trabalho. Junto a isso tive problemas pessoais. Fiz um acordo e me desliguei da empresa." Agora, a jovem diz que está bem. Ela contou que ainda toma ansiolíticos, para diminuir a ansiedade, mas está reduzindo gradativamente a dosagem. (A Tribuna-17/07/2015)

A notícia, ainda que de forma indireta, poderia estimular os sujeitos ao uso de medicamento, em especial os ansiolíticos, conforme o próprio título salienta: 'Remédios mais eficazes são apostas de médicos para tratar também pacientes com diabetes, câncer e doença pulmonar', no entanto, evidencia-se uma lacuna e dúvidas sobre a intenção da notícia.

De acordo com Ferraz (2014), a medicalização da vida garante forças necessárias para dar continuidade ao 'corpo máquina' como parte da engrenagem para o trabalho, que não mais escuta e não aceita seus limites. Deve-se ressaltar que a invenção de novas doenças traz a possibilidade de novos mercados de medicamentos com o consentimento aberto da mídia. Nesse caso, Barros (2014), Catalan-Matamoros, Pariente e Elías-Pérez (2019) discutem o fenômeno da medicalização e o papel da mídia quanto ao uso de medicamentos, e também os impactos das notícias na ampliação de usos indevidos.

Verifica-se que o caso noticiado relata o desligamento da trabalhadora da empresa como solução do problema. Essa ideia induz os leitores a vislumbrarem uma solução factível para a maioria, desconsiderando a realidade econômica e social do país, que tem um histórico de enormes desigualdades de oportunidades no mundo do trabalho, com uma taxa de desemprego atual elevada, chamada taxa de subutilização, que foi de $25 \%$, representando 28,3 milhões de pessoas no primeiro trimestre de 2019 (IBGE, 2019). Conforme alerta Dedecca (2005), a maior parte da população dos países emergentes encontra-se em más condições de trabalho, desemprego ou trabalho informal. Mesmo que o trabalhador sinta o desejo de abandonar o emprego ou que esteja em sofrimento, na maioria das vezes, ele não tem essa opção, pela necessidade básica de sobrevivência, o que o leva ao adoecimento. De acordo com Dejours $(1992,2007)$, existem mecanismos de defesa do trabalhador que per- 
mitem que ele sobreviva ao sofrimento imposto pela organização do trabalho vigente, desconsiderando sua história de vida, seus desejos e motivações. No entanto, a busca pelo lucro e pelo aumento da produção nas organizações não isenta a produção de problemas sociais e humanos.

Partindo para uma abordagem geral, o estresse no trabalho é uma das fontes mais comuns de doenças e estimativa de morte na Europa (European Agency for Health and Safety at Work, 2009), além dos riscos psicossociais (Ganster e Rosen, 2013), cenário que se repete na América Latina, América Central e África (Steyn e Vawda, 2014), e em outras partes do mundo (Hausmann et al., 2014). No Brasil, o aumento do estresse ocupacional tem relação entre capital e trabalho, o que acentua as desigualdades sociais (Rodrigues et al, 2017), dificultando o acesso ao emprego (Martins e Molinaro, 2013), e contribuindo para a privação dos direitos fundamentais do homem (Coelho, Tapajós e Rodrigues, 2010). Esses aspectos do estresse ocupacional também estão relacionados à terceira revolução industrial, com a entrada as novas tecnologias (Braga, Carvalho e Binder, 2010). Por isso, o estresse é um fenômeno da contemporaneidade que goza de uma indefinição conceitual quanto à classificação de doenças ou de fator de risco para doenças, apesar de possuir CID. Cavaca, AG (2015) e Garcia et al. (2019) destacam que entre as doenças mais noticiadas no Espírito Santo (ES), o'agravo', 'reações ao stress grave e transtornos de adaptação', ocupa a sexta posição entre as mais noticiadas, que significa no CID 10, "acontecimento particularmente estressante que desencadeia uma reação de estresse aguda que em decorrência de fatores desagradáveis e duradouros, levam a um transtorno de adaptação" (Departamento de Informática do Sistema Único de Saúde - Datasus, 2008, p.10). Enfatiza-se a distância entre prioridade epidemiológica e os critérios de produção noticiosa, a mídia lida com as problemáticas da saúde como produtos comercializáveis, às vezes, sem preocupação com o fator bem-estar. No entanto, temos que levar em conta também as condições de produção da notícia e a natureza do dispositivo jornalístico como um campo que produz um discurso público para um público universal e não específico.

Quanto ao tempo (horas de trabalho) de execução da tarefa, os horários podem ser rigorosos, longos, inflexíveis e até mesmo antissociais, visto que interferem nos ciclos circadianos, ritmos biopsicossociais, metabólicos e orgânicos (James et al., 2017), aliado a um ambiente organizacional ineficiente e inadequado quanto ao espaço do trabalho. As jornadas de trabaIho têm sido apontadas como um dos riscos de estresse mais enfatizados pelos trabalhadores (Oliveira, Silva e Lima, 2018). Essa constatação pôde ser verificada nas notícias analisadas: 
(...) senso comum que trabalhar demais não faz bem à saúde, mas o estresse não é a única doença provocada pelo excesso de trabalho. Pesquisa divulgada ontem pela revista The Lancet, da Inglaterra, mostrou que trabalhar 55 horas ou mais por semana aumenta em 33\% o risco de enfarte (A Tribuna-21/08/2015).

Um estudo norte-americano descobriu que o índice de suicídios entre médicos é $70 \%$ maior do que na população em geral. Entre médicas, o índice aumenta em $400 \%$. Especialistas apontam que fatores como ansiedade, depressão, alto número de horas trabalhadas, estresse e responsabilidade em lidar com tragédias humanas podem explicar tal fato. (A Tribuna-10/03/2016).

Alguns autores enfatizam que as notícias positivas podem potencializar e promover mudanças de comportamentos saudáveis de forma mais efetiva do que destacar os efeitos negativos dos comportamentos sem dar soluções (Gallagher e Updegraff, 2012; Covey, 2014). Em discordância desses argumentos, observa-se que grande parte dos jornalistas apresenta uma postura que não contribui positivamente para a saúde dos trabalhadores, visto que muitas vezes o enfoque prioritário está nos efeitos negativos decorrentes do trabalho. Isso foi observado no enquadramento negativo da saúde na notícia citada, que não forneceu alternativas ao trabalhador para essa situação. As notícias trazem estatísticas retiradas de estudos científicos de impacto sem uma contextualização, não cumprindo assim a finalidade social de esclarecimento e orientação, que seria o papel da imprensa, esperado pela sua função social.

Nesse sentido, Luiz (2006) conceitua as diferentes abordagens de comunicação da ciência, como a comunicação midiática. Segundo esse autor, a mídia educa e deseduca a população, influenciando o seu comportamento, a sua atitude ou a formação da sua opinião, baseada no que define como prioridade do jornal no dia 'ordem do dia' ou como 'agenda' dando ênfase ou 'visibilidade', ou deixando lacunas 'invisibilidade' ou distorcendo fatos sobre os riscos epidemiológicos. Em adição, Shoemaker e Cohen (2006) descrevem sobre a variabilidade de eventos noticiosos significativos no exercício do jornalismo e concluem que, apesar das notícias variarem em todas as partes do mundo, a natureza delas se repete e as pessoas têm noções semelhantes a respeito da notícia.

Outro ponto que se relaciona à carga, ritmo e horas trabalhadas é a falta de métodos, autonomia e participação nas tomadas de decisões e controle (participação e controle) sobre o ambiente ocupacional que intensificam os riscos. Assim, Lua et al. (2018) descrevem sobre avaliação negativa do trabalho da enfermagem diante dos efeitos do estresse; Dejours (2008) destaca o caso dos médicos, que algumas vezes não têm como saber se as 
falhas na sua profissão estão relacionadas à sua incompetência ou a anomalias técnicas, o que é fonte de angústia para esse profissional. Como por exemplo no trecho a seguir: "Médico - Desafios: trabalhos por escala e falta de condições de atendimento, principalmente em hospitais públicos, são fatores de estresse." (A Tribuna-09/10/2016)

Essa notícia aborda algumas profissões e cita os desafios relacionados a cada uma delas. No caso do profissional médico, ressalta-se o trabalho por escala e a falta de condições de atendimento. Entende-se que os profissionais de saúde, muitas vezes, não têm controle sobre os métodos, ritmo, horas e ambiente de trabalho, conforme nesse trecho:

Trabalhei 29 anos como servidor da UFES [Universidade Federal do Espírito Santo]. Uma parte foi como médico na UTI [unidade de terapia intensiva] neonatal, do Hospital das Clínicas, onde, por estresse, tive quatro hérnias de disco. (A Gazeta-23/08/2016)

A notícia enfatiza os problemas biológicos, assim como Lerner (2014) discute os procedimentos da imprensa no contexto da cobertura dos assuntos de saúde como um fenômeno ligado ao lugar que a saúde vem ocupando na sociedade contemporânea, isto é, ainda bastante centrado nos aspectos biológicos, desconsiderando os aspectos culturais, históricos e sociais. Sob esse aspecto, Friedman, Tanner e Rose (2014) fizeram um estudo sobre as percepções dos jornalistas para avaliar as informações sobre a saúde e relatam que buscam manchetes fortes e histórias pessoais que causem impacto na população. Além disso, afirmaram que as notícias são bastante técnicas, até não inapropriadas para o público do jornal, e indicaram que o jornalismo de saúde poderia ser melhorado. Nesse sentido, a mídia teria capacidade de ser uma grande aliada do sistema de saúde, e esse envolvimento poderia ser útil para os formuladores de políticas de saúde (Wang et al., 2017).

\section{Contexto dos riscos do estresse: desenvolvimento de carreira, status e pagamento, função na organização, as relações interprofissionais, a cultura organizacional e a interface trabalho-família}

A reflexão sobre o desenvolvimento de uma carreira profissional relacionada à qualificação se depara também com vários aspectos que vão impactar fortemente o trabalhador em sua saúde e qualidade de vida. Assim, situações relativas ao salário, status proporcionado pela ocupação, falta de perspectivas promocionais, instabilidade no emprego, forma pela qual o pagamento é realizado, ou seja, se por produtividade, por exemplo, e 
ainda escassez ou mesmo inexistência de qualificação profissional, bem como formas de avaliação poucos claras ou mesmo injustas, isso quando existentes, são fatores que influenciam diretamente nesse quesito.

De acordo com Dejours (1992), na organização do trabalho, outras variáveis que dependem do prazer e desprazer do trabalhador estão relacionadas, como o sistema hierárquico, as lideranças, as relações de poder, o grau de segurança e as responsabilidades atribuídas a cada trabalhador, as quais são exemplificadas nos trechos:

Os motoristas que atuam nos sistemas de ônibus (...) passam por curso de formação de 50 horas (...), no curso eles aprendem conteúdos de direção defensiva, legislação de trânsito, relacionamento interpessoal e primeiros socorros (...) passam por reciclagens periódicas, (...) é necessária a renovação anual de um curso de 18 horas, onde são atualizadas as noções de legislação e condutas no trânsito (...)“Houve redução dos carros em várias linhas e os ônibus andam lotados. Isso causa estresse e alguns motoristas andam mais rápido para cumprir os horários e tentar ter algum descanso", afirmou um rodoviário do sistema (...) (A Tribuna-27/08/2016)

Nessa notícia percebe-se que além do estresse do motorista, é colocada em risco a vida dos passageiros. No cenário de aceleração, as situações podem exceder a capacidade do trabalhador para equilibrar demandas e desafios, então, ele passa a correr riscos em sua saúde, podendo acarretar falhas na segurança da organização. Para minimizar esses riscos, são identificadas associações entre apoio organizacional e estresse ocupacional em trabalhadores (Zeytinoglu et al., 2017). Além disso, dentro das abordagens para o estudo dos riscos psicossociais do trabalho, o estresse relacionado ao trabalho é a dimensão central de pesquisas em segurança e saúde ocupacional (Milczarek, Schneider e Rial González, 2009). O estresse favorece o aumento de incidência de acidente ocupacional (Rotta et al., 2018).

A notícia citada enfatiza a conexão entre qualificação profissional e segurança no trabalho, e aponta esse problema de forma completa, uma vez que descreve o problema da redução da frota de ônibus e alerta a população para os riscos decorrentes dessa tomada de decisão por parte dos operadores do transporte coletivo. Além disso, a notícia expõe o nome da empresa, o que facilita a fiscalização para o processo de segurança, e destaca o número de telefone para reclamações, além de incluir a fala dos passageiros. A mídia faz uma cobertura completa do fenômeno da realidade social e contribui para que a população busque lutar pelos seus direitos de segurança; dessa forma, auxilia para que a população se conscientize das suas possibilidades. Isso permite que a prestação de serviços na sociedade 
seja mais equilibrada, proporcionando indiretamente que os trabalhadores tenham mais satisfação na função. Nessa notícia específica verifica-se que a mídia conseguiu cumprir seu papel social e dar voz aos sujeitos sociais.

Em relação à função na organização, destaca-se a relação intensa e contínua com o público, a sobrecarga de responsabilidades e até mesmo papéis pouco claros e definidos para determinadas funções. A notícia a seguir destaca a dificuldade de lidar continuamente com pessoas e seus problemas, conforme ilustra o trecho:

Um dos profissionais lida com a saúde das pessoas e o outro, com a educação. Os dois deveriam ser valorizados, (...) médicos e professores são duas profissões que passam por um alto nível de estresse. Com isso, problemas como depressão, ansiedade e sensação de impotência pode[m] aparecer,(..). "Temos a estimativa de que $90 \%$ das vezes que ocorre essa ameaça são no serviço público" (...) “O estresse pode ir para vários caminhos, como ter rejeição ao ambiente de trabalho, ficar desmotivado e até ter momentos de crise aguda no ambiente de trabalho. É preciso buscar ajuda." (A Tribuna-13/05/2015).

No recorte temporal adotado neste estudo, fica evidenciado que a mídia noticiou sobre a saúde pública fazendo enquadramentos de episódios relacionados ao Sistema Único de Saúde e escolas públicas com tendência a dar visibilidade às notícias negativas, semelhante ao que foi verificado em outros estudos sobre o sistema de saúde brasileiro nos meios de comunicação (Ortona e Fortes, 2012; Machado, 2014; Silva et al., 2018; Langbecker, Castellanos e Catalan-Matamoros, 2019).

Araújo e Cardoso (2007) reiteram que a mídia pode exercer o papel de transformador da realidade da saúde. No entanto, os estudos demonstram que a mídia tem enfatizado e dado mais visibilidade às fragilidades do sistema público, sem apresentar seus pontos fortes e suas potencialidades. Além disso, há, embutidos nos jornais, espaços privilegiados de divulgação de venda dos serviços privados como planos de saúde e outros serviços e produtos. Em seu estudo, Cavaca, AG et al. (2016) demonstram que algumas doenças no espaço midiático são negligenciadas e outras têm visibilidade de forma descontextualizada das necessidades da realidade local. Oliveira (2000) também ressalta a forma como a mídia interpreta e retrata o campo da saúde na perspectiva das relações de poder, dando realce aos acontecimentos desfavoráveis do sistema público e aos favoráveis do privado.

Nessa discussão, Silva (2014) descreve sobre os critérios das notícias, como são escolhidas e quais são elegíveis no percurso entre a construção e a seleção da notícia no Brasil. Na mesma direção, Emerich et al. (2018) 
retratam sobre as características das indústrias culturais e midiáticas e destacam que os anúncios são muitas vezes responsáveis por sustentar o setor do jornalismo, além de sinalizar a posição ideológica, comercial e política da publicação.

Constata-se ainda que os anúncios e propagandas publicadas nos jornais sobre os planos privados são ilustrados com pessoas 'felizes,' 'sorrindo' e 'bem-sucedidas'. As fotos retratam pessoas de classe alta, com seus belos carros e roupas elegantes em praias e montanhas paradisíacas. Segundo Oliveira (2014), o interesse da mídia jornalística está diretamente relacionado com o crescimento da oferta de bens e serviços, cuja produção simbólica sugere uma vida bem-sucedida no que se refere a bens materiais e inclusão em serviços privados.

Outro ponto a ser explorado é o conflito inerente e existente em certos ambientes de trabalho, que também desempenha um papel crucial e que contribui para a compreensão dos riscos do estresse ocupacional, os quais variam entre pares de trabalho, gerência e outros.

As relações interprofissionais, entre os pares, contistuem-se em outros agravantes quando essas relações se tornam imprudentes, baseadas em fracos relacionamentos entre colegas, em especial quando há intimidação, violência e assédio, ou mesmo nos casos em que uma determinada ocupação, ao contrário, dá-se em um ambiente isolado e solitário. Os ambientes relacionais hostis tendem a ter mais problemas de saúde, mesmo que os recursos materiais sejam ideais (Dejours, 1992; Schyns e Van Veldhoven 2010; Füllemann et al., 2016). Os trechos a seguir são representativos dessa classificação:

Estresse gerado: "A pessoa fica mal-humorada, perde a produtividade pois está reclamando o tempo todo, tirando o foco da resolução dos problemas. Esse estresse passa para o colega do lado e afeta a relação pessoal. No final das contas, as empresas cobram resultados e só se queixar torna esse colaborador um sério candidato a ser demitido" (A Gazeta-23/10/2016).

Essa notícia destaca que além de interferir negativamente no trabalho do grupo, esse trabalhador mal humorado, e que reclama frequentemente, é um candidato à perda do emprego, dada sua postura negativa, que passa a afetar o conjunto dos trabalhadores. A notícia, nesse caso específico, constrói a ideia de que o trabalhador que é o vilão, culpabilizando-o por ser um desagregador. Observa-se, ainda, que esse discurso midiático transmite uma ideologia voltada às elites simbólicas, abordando os problemas desvinculados do contexto sociocultural, histórico e político, e, desse modo, 
reproduz uma nova situação de exclusão (Van Dijk, 2000; Thompson, 2007; Carvalho, 2008; Medeiros e Guareschi, 2008; Charaudeau, 2009).

Segundo Garbin e Fischer (2012) a mídia jornalística tende a naturalizar o fenômeno do assédio moral acentuando o caráter individualista e minimizando uma abordagem coletiva, propagando o tema em uma perspectiva estigmatizante e banalizada da violência no trabalho. Isso está presente no âmbito das relações interprofissionais, mas pode ser resultado de uma cultura organizacional que não coíbe essas práticas estressoras.

Com relação à cultura organizacional há uma relação direta com a estrutura da organização e sua forma de comunicação, interelacionando e potencializando estes fatores de riscos de estresse de forma substantiva. Nesse sentido, a existência de lideranças negativas e falta de clareza de objetivos a serem alcançados pela organização, aliado à inadequação, à existência de equipamentos inapropriados e à inadequação da estrutura física, seja ela espacial ou de acessibilidade, são os fatores que se colocam em destaque na discussão deste item, como se pode ilustrar nos trechos seguintes:

São diversos os aspectos que distinguem um ambiente organizacional de uma empresa. Entre eles estão as condições adequadas de trabalho, o clima dentro da empresa, se há omissão ou se os profissionais são reconhecidos como peças fundamentais para a instituição. De acordo com a diretora de Desenvolvimento de Talentos, Liderança e Engajamento da consultoria em mobilidade de talentos (...), hoje os profissionais estão trabalhando mais e num cenário de estresse e pressão muito alto, o que vem provocando inúmeros problemas entre os trabalhadores. (A Tribuna-03/05/2015)

Essa notícia traz elementos importantes para que o trabalhador se localize na estrutura de processo de trabalho. No entanto, cria-se uma distância entre ele e a notícia quando se coloca um profissional (diretor de Desenvolvimento de Talentos) pouco conhecido para falar do tema. Além disso, a narrativa jornalística ignora as características sistêmicas, estruturais e sociais ao analisar o risco à saúde relacionado aos problemas inter-relacionais e não apresenta perspectivas de soluções, revelando que a orientação de carreira afeta adequadamente o desempenho dos funcionários e ajuda a desenvolver novas abordagens e soluções para os problemas (Van Dijk, 2000; Thompson, 2007; Carvalho, 2008; Medeiros e Guareschi, 2008; Charaudeau, 2009). Nesse contexto, Niemann et al. (2015) também apresentam que o feedback nas organizações pode colaborar para uma melhoria no desempenho de trabalhadores e organizações. 
Outro ponto a ser destacado é a interface trabalho e família, que também se constitue numa relevante fonte de conflitos, que pode ativar causas que vão afetar sobremaneira o estresse profissional. A instabilidade gerada por uma escala de trabalho não regular implica em estabelecer uma convivência irregular com a familia e naturalmente um funcionamento conflituoso, como sugere o trecho a seguir:

"A babá (...) trabalhou (...) na casa da psicóloga (...) Muitos pais acabam não dando a atenção necessária aos filhos, por conta do estresse do trabalho (...)" (A Tribuna 24/07/2016)

No período deste estudo, esta foi a única notícia encontrada relacionando trabalho e família. Percebe-se que a notícia completa não traz nenhuma informação educativa ou difusão de conhecimento que beneficie o leitor, não atribui sentido à leitura para que possibilite ao trabalhador reflexão e outras interpretações (Cavaca, AG et al., 2012; Setton, 2011). Evidencia-se também que, dentro da perspectiva do trabalho, no olhar da mídia, pouca preocupação tem sido conferida ao tema da família e estresse no trabalho.

Como reflexão geral, observa-se que no dia da publicação desta notícia foram editadas 14 páginas que discorreram sobre o tema estresse, o que reafirma o fato de estar em alta no contexto do trabalho, inclusive na chamada da capa principal do jornal "Mutirão contra estresse nas empresas". No entanto, as notícias jornalísticas não parecem ser expositivas, com apresentação de fatos de importância e relevância, mas sim textos noticiosos, que se enquadraram como narrativas e como discursos (Silva, 2007), que são pouco capazes de fomentar a participação dos trabalhadores como sujeitos ativos para a redução do estresse e promoção da saúde.

As notícias, muitas vezes, aparecem como entretenimento entre catástrofes e eventos sociais para preencher a necessidade de fantasia do leitor sem levar uma informação útil que o potencialize e estimule seu protagonismo. De acordo com Spink (1993, 2019), o ser humano é resultado daquilo que consome; e nesse caso, o leitor, que é o trabalhador, está consumindo notícias que servem como entretenimento ou até o alienam. No entanto, ressalta-se que a análise de conteúdo nunca pode se ater exclusivamente ao que foi publicado pelo jornal, mas também às condições de produção da notícia e à natureza do dispositivo jornalístico, como um campo que produz um discurso público, para um público universal, sem especificidades (Rodrigues, 1997, 2016).

No recorte temporal deste estudo, para desenvolver o tema do estresse ocupacional, a mídia parece não ter ampliado a reflexão e a busca de soluções para o trabalhador, bem como não esclareceu sobre as legislações 
e direitos dos trabalhadores. No que se refere à abordagem de qualidade na prevenção do estresse ocupacional que combina conteúdo e processo, Kompier, Cooper e Geurts (2000) descrevem sobre o assunto e sobre gerenciamento para o novo milênio (Richardson, 2017). Uma das estratégias de prevenção poderia ser a inserção da mídia para dar visibilidade à legislação vigente e uma comunicação mais interativa sobre o trabalho.

Observou-se ainda que grande parte das notícias de estresse ocupacional foi sobre categorias profissionais: médicos, professores e militares. Os entrevistados foram principalmente profissionais de saúde e educação; profissionais de nível superior ou de alto cargo, como executivo, secretário de segurança, político, celebridade no mundo artístico. Houve pouca visibilidade de atividades como pedreiro, empregada doméstica, lavrador, trabalhador do comércio; e a participação da população em geral foi imperceptível.

Além de abordar principalmente conteúdos negativos sobre o sistema público, a maior parte das pessoas citadas são celebridades e políticos, as notícias são repetitivas e muitas vezes não acrescentam nenhuma novidade ao repertório do leitor. Percebeu-se ainda que algumas notícias foram copiadas dos grandes jornais. Além disso, algumas notícias estavam com algum comercial de produtos e serviços embutido e apresentaram uma ilustração do produto, mercadoria ou serviço para chamar atenção do trabalhador.

Em relação às intervenções sobre os riscos do estresse ocupacional, no sentido da promoção de saúde e prevenção de doença, a literatura científica tem avançado progressivamente (Leka, Griffiths e Cox, 2004; Zavalis, 2015; Moraes Filho e Almeida, 2016; Håkansson e Ahlborg, 2018). No entanto, a mídia jornalística encontra grandes lacunas, segundo Monteiro e Marques-Pinto (2017) a profissão de jornalistas também gera muito estresse, tanto pelo estresse do cotidiano de trabalho, quanto pelos eventos dramáticos a que estão submetidos. As falhas do jornalismo, no que se refere ao conhecimento sobre o conceito de promoção de saúde, visto que as notícias visibilizaram principalmente o estresse como doença ou como fator de risco para outras doenças, está na falta de especialização do profissional da comunicação sobre o tema saúde. Percebeu-se que houve poucos relatos do desprazer no trabalho e sobre os desdobramentos da organização no trabalho (Dejours, 1992), que gera sofrimento (Dejours, 1993), que potencializa o estresse ocupacional. A mídia deveria estimular ações promotoras de saúde, por meio das notícias. De acordo com Marcelino Filho e Araújo (2015), deve-se pensar em programas de proteção, valorização e promoção à saúde dos trabalhadores visando à qualidade de vida no trabalho. 


\section{Considerações finais}

Este estudo discutiu o estresse ocupacional na mídia jornalística impressa, sob a ótica da organização do trabalho (Dejours, 1992), entendendo que esse tipo de informação pode fortalecer e protagonizar o trabalhador ou, ao contrário, alienar e enfraquecê-lo. Constatou-se que, para potencializar o trabalhador e diminuir o sofrimento relacionado ao trabalho, serão necessárias notícias mais problematizadoras, mais interativas e com cunho esclarecedor sobre direitos do trabalhador.

Verificou-se também que as notícias de cunho relacional foram as que apresentaram menor realce, ao contrário das notícias alarmantes a respeito dos riscos de estresse ocupacional que tiveram destaque. Além disso, as notícias sobre prevenção de doenças e promoção de saúde obtiveram pouca visibilidade, assim como no que tange a uma problematização de legislações trabalhistas. Nesse sentido, Dejours (1992) defende o empoderamento dos trabalhadores a respeito da importância da saúde mental, física e social, na tentativa de minimizarem os riscos do estresse ocupacional.

Além do mais, percebe-se a possibilidade da contribuição da mídia impressa para potencializar a difusão da legislação e dos direitos do trabaIhador em seus espaços de trabalho, fortalecendo as pautas de lutas por condutas mais humanizadas da organização, garantindo mais qualidade de vida e saúde, além do aumento do rendimento geral da produtividade no seu âmbito global.

Nessa direção, a análise geral sobre as notícias sobre estresse ocupacional na mídia jornalística impressa indica que há contradições peculiares à vida social, no sentido de que há visibilidade da ideologia, dos costumes e dos valores da classe dominante, influenciando o trabalhador com crenças voltadas a um público seleto. $O$ estudo revelou, ainda, que prevalece a reprodução de aspectos negativos e estereótipos do bom trabalhador como aquele que não reage contra os abusos das organizações, situações cotidianas de sofrimento que marcam sobremaneira a vida dos trabalhadores com o estresse e suas consequências em longo prazo.

Verificou-se também que a violação de direitos do trabalhador tem uma abordagem de invisibilidade e, em contrapartida, as notícias de cunho economicistas e mercadológicas sobre os efeitos do estresse do trabalhador têm sido enfatizadas. Assim, há a necessidade premente de uma reflexão acadêmica e midiática a respeito das interconexões dos riscos do estresse no trabalho - e as doenças provenientes - e a amplificação do estresse como fator de risco na sociedade contemporânea. 


\author{
ESTRÉS OCUPACIONAL EN LOS MEDIOS IMPRESOS: \\ UNA PERSPECTIVA DE CHRISTOPHE DEJOURS
}

\begin{abstract}
Resumen El estrés en el trabajo afecta los aspectos psicológicos, físicos y sociales del trabajador, así como la eficacia de las organizaciones productivas. El objetivo de este estudio fue comprender el estrés ocupacional en los medios periodísticos bajo la perspectiva de la organización del trabajo. Se trata de una pesquisa cualitativa conducida por análisis de contenido de Bardin, con base en las categorías analíticas previamente definidas por la Organización Mundial de la Salud, en el informe "Work Organization \& Stress", bajo la perspectiva del referencial teórico de Christophe Dejours. Han sido clasificadas 727 noticias que tenían como palabra clave "estrés", siendo 130 noticias específicas sobre el tema del estrés en el trabajo. Estas noticias han sido clasificadas en dos categorías, cada cual con subcategorías: "Contenido de los Riesgos del Estrés" (47) y "Contexto de los Riesgos del Estrés" (59). Se constató que los medios ya casi no funcionan como portavoces de los trabajadores con el objetivo de cumplir su papel social, sin estimularlos como sujetos sociales. Se propone que los medios periodísticos estimulen acciones que promuevan el protagonismo de los trabajadores, con base en la visibilidad de las legislaciones vigentes, e indiquen caminos más directos para que los trabajadores puedan luchar por la garantía de derechos a la salud en el combate al estrés, con perspectiva crítica del entendimiento sobre la organización del trabajo y sus potencialidades en la promoción de la salud.
\end{abstract}

Palabras clave medios de comunicación; estrés ocupacional; jornada laboral

\title{
Colaboradores
}

Átala Lotti Garcia participou da concepção e desenho do estudo, análise dos dados e redação do manuscrito, participação dos resultados e discussão e da revisão final. Tatiana Breder Emerich participou na análise dos dados, redação do manuscrito e revisão final. Luciane Bresciani Salaroli participou na redação do manuscrito, revisão final. Aline Guio Cavaca participou na concepção do projeto, redação do manuscrito e revisão final. Edson Theodoro dos Santos Neto colaborou na interpretação dos dados, redação do manuscrito e aprovação da versão final.

\section{Financiamento}

Não houve. 


\section{Referências}

ALBUQUERQUE, Guilherme S. C. et al. Exploração e sofrimento mental de professores: um estudo na rede estadual de ensino do Paraná. Trabalho, Educação e Saúde, Rio de Janeiro, v. 16, n. 3, p. 1.287-1.300, dez. 2018.

ARAÚJO, Inesita S.; CARDOSO, Janine M. Comunicação e saúde. Rio de Janeiro: Editora FIOCRUZ, 2007.

AREDES, Janaína S.; GIACOMIN, Karla C.; FIRMO, Josélia O. A. A práxis médica no pronto atendimento diante do paciente com sequelas crônicas: culpa, temor e compaixão. Trabalho Educação e Saúde, Rio de Janeiro, v. 16, n. 3, p. 1.177-1.199, dez. 2018.

BARROS, José A. C. A dicotomia entre ciência e negócios, a mídia e a medicalização na sociedade contemporânea (PARTE 2). Boletim informativo Geum, Piauí, v. 5, n. 3, p.79-93, jul./set. 2014.

BAO, Stephen S. et al. Relationships between job organizational factors, biomechanical and psychosocial exposures. Ergonomia, Cambridge, v. 59, n. 2, p. 179-94, 2016.

BARDIN Lawrence. Análise de conteúdo. São Paulo: Edições 70, 2015. 288 p.

BRAGA, Ludmila C.; CARVALHO, Lidia R.; BINDER, Maria C. P. Condições de trabalho e transtornos mentais comuns em trabalhadores da rede básica de saúde de Botucatu (SP). Ciência e Saúde Coletiva, Rio de Janeiro, v. 15, p. 1.585-1.596, Jun. 2010. Suplemento 1.

CARVALHO, Mónica. Comunicação, medicina e evolucionismo: estudo de caso dos media brasileiros. Observatório $\left(\mathrm{OBS}^{*}\right)$, Lisboa, v. 2, n. 4, p. 311-329, 2008.

CASTELL, Sarah et al. Public attitudes to science 2014: main report. Londres: Department for business, innovation and skills, 2014. Disponível em: <https://assets.publishing.service.gov.uk/ government/uploads/system/uploads/attach- ment_data/file/348830/bis-14-p111-public-attitudes-to-science-2014-main.pdf $>$. Acesso em: 9 set. 2019.

CATALAN-MATAMOROS, Daniel; PARIENTE, Antoine; ELÍAS-PÉREZ, Carlos. What we know about media communication on antibiotics and antimicrobial resistance: a systematic review of the scientific literature. Patient Education and Counseling, Oslo, v. 102, n. 8, p. 1.427-1.438, mar. 2019.

CAVACA, Aline G. et al. As representações da saúde bucal na mídia impressa. Interface: Comunicação, Saúde, Educação, Botucatu, v. 16, n. 43, p. 1.055-1.068, 2012.

CAVACA, Aline G. Doenças midiaticamente negligenciadas: cobertura e invisibilidade de temas sobre saúde na mídia impressa. 2015. 177f. Tese (Doutorado em Saúde Pública)-Escola Nacional de Saúde Pública Sergio Arouca, Rio de Janeiro, 2015.

CAVACA, Aline G; EMERICH, Tatiana B; LERNER Kátia. Observatórios de Saúde na Mídia: dispositivos de análise crítica em Comunicação e Saúde [editorial]. Revista Brasileira de Pesquisa em Saúde, Vitória, v.18, n.3, p. 4-5, 2016.

CAVACA, Aline G. et al. Diseases neglected by the media in Espírito Santo, Brazil in 2011-2012. PLOS Neglected Tropical Diseases, San Francisco, v. 10, n. 4, 2016.

CAVACA, Sthefany D. et al. Observatório de Saúde na Mídia - Regional Espírito Santo: relato de uma experiência interdisciplinar em Saúde Coletiva. Revista Brasileira de Pesquisa em Saúde, Vitória, v. 20, n. 2, p. 149-156, abr-jun, 2018.

CHARAUDEAU, Patrick. Discurso das mídias. São Paulo: Contexto, 2009.

COELHO, Maria F. P.; TAPAJÓS, Luziele M. S.; RODRIGUES, Monica. Políticas sociais para o desenvolvimento: superar a pobreza e promover 
a inclusão. Brasília: Ministério do Desenvolvimento Social e Combate à Fome, UNESCO, 2010.

COHEN, Sheldon; GIANAROS, Peter J.; MANUCK, Stephen B. A Stage Model of Stress and Disease. Perspectives on Psychological Science, v. 11, n. 4, p. 456-463, 2016.

COQUEIRO, Jandesson M. et al. Diabetes mellitus na mídia impressa: uma proposta de protocolo de coleta e classificação de dados para pesquisa. Revista Brasileira de Pesquisa em Saúde, Vitória, v. 20, n. 2, p. 74-87, 2018.

COTÉ, Stéphane. A social interaction model of the effects of emotion regulation on work strain. Academy of Management Review, New York, v. 30, n. 3, p. 509-530, 2005.

COVEY, Judith. The role of dispositional factors in moderating message framing effects. Health Psychology, Cambridge, v. 33, n. 1, p. 52-65, 2014.

DANNA, Karen; GRIFFIN, Ricky W. Healthy and well being in the workplace: A review and synthesis of the literature. Journal of Management, Texas, v. 25, n. 3, p. 357-384, 1999.

DEDECCA, Cláudio S. Notas sobre a evolução do mercado de trabalho no Brasil. Revista de Economia Política, São Paulo, v. 25, n. 1 (97), p. 113-130, janeiro-março, 2005.

DEJOURS, Christophe. A loucura do trabalho: estudo da psicopatologia do trabalho. 5. ed. ampl. São Paulo: Cortez; Oboré, 1992. 168p.

DEJOURS, Christophe. Uma nova visão do sofrimento humano nas organizações. In: CHANLAT, Jean-François. O indivíduo na organização: dimensões esquecidas. 3. ed. São Paulo: Atlas, 1993. p. 149-174. Volume 1.

DEJOURS, Christophe. A carga psíquica do trabalho. In: DEJOURS, Christopher; ABDOUCHELI, Elisabeth; JAYET, Christian. Psicodinâmica do trabalho: contribuições da escola dejouriana à análise da relação prazer, sofrimento e trabalho. São Paulo: Atlas, 1994a. p. 21-32.

DEJOURS, Christophe; ABDOUCHELI, Elisabeth. Itinerário teórico em psicopatologia do trabaIho. In: DEJOURS, Christopher; ABDOUCHELI,
Elisabeth; JAYET, Christian. Psicodinâmica do trabalho: contribuições da escola dejouriana à análise da relação prazer, sofrimento e trabalho. São Paulo: Atlas, 1994b. p. 87-99.

DEJOURS, Christophe. A banalização da injustiça social. 7. ed. Rio de Janeiro: Ed. FGV, 2007. 160 p.

DEJOURS, Christophe. Da psicodinâmica à psicopatologia do trabalho. In.: LANCMAN, Selma; SZNELWAR, Laerte I. (org.). Rio de Janeiro: Editora Fiocruz; Brasília: Paralelo 15, 2008. p. 1-512.

DEPARTAMENTO DE INFORMÁTICA DO SISTEMA ÚNICO DE SAÚDE (Datasus). Trata-se da décima Classificação Estatística Internacional de Doenças e Problemas relacionados à Saúde, publicada pela Organização Mundial da Saúde (OMS), categoria única (código CID-10), 2008. Disponível em: <http://www.datasus.gov.br/cid10/ V2008/apresent.htm> Acesso em: 01 abril 2019.

EMERICH, Tatiana B. et al. O jornal folha de S. Paulo no contexto das indústrias culturais e midiáticas: um olhar para as manchetes sobre o Programa Mais Médicos. Revista Brasileira de Pesquisa em Saúde, Vitória, v. 20, n. 2, p. 16-24, abr./jun. 2018.

ERTEL, Michael et al. Adverse psychosocial working conditions and subjective health in freelance media workers, Work \& Stress, Netherlands, v. 19, v. 3, p. 293-299, 2005.

\section{EUROPEAN AGENCY FOR HEALTH AND SAFETY} AT WORK. OSH in figures: stress at work: facts and figures. Luxembourg: Office for Official Publications of the European Communities, 2009. (European Risk Observatory Report, 9) Disponível em: <https://osha.europa.eu/en/ tools-and-publications/publications/reports/ TE-81-08-478-EN-C_OSH_in_figures_stress_ at_work>. Acesso em: 9 set. 2019.

FAN, Libra B. et al. Work and home stress: associations with anxiety and depression symptoms. Occupational Medicine, Oxford, v. 65, n. 2, p.110-116, mar. 2015.

FERRAZ, Luiz M. R. Entre remédios e hábitos saudáveis: a medicalização nos discursos de Veja e Época. In: LERNER, Kátia; SACRAMENTOm 
Igor. Saúde e jornalismo: interfaces contemporâneas. Rio de Janeiro: Fiocruz, 2014, p. 219-234.

FRIEDMAN, Daniela B.; TANNER, Andrea; ROSE India D. Health journalists'perceptions of their communities and implications for the delivery of health information in the news. Journal of Community Health, New York, v. 39, n. 2, p. 378-385, 2014.

FÜLLEMANN, Désirée et al. Individual and grouplevel job resources and their relationships with individual work engagement. Journal of Occupational Health, Japan, v.58, n.3, p.255-268, 2016.

GALLAGHER, Kristel M.; UPDEGRAFF, John A. Health message framing effects on attitudes, intentions, and behavior: A meta-analytic review. Annals of Behavioral Medicine, Oxford, v. 43, n. 1, p. 101-116, 2012.

GANSTER, Daniel C; ROSEN, Christopher C. Work stress and employee health: a multidisciplinary review. Jornal of Management, Texas, v. 39, n. 5, p.1.085-1.022, 2013.

GARBIN, Andréia C.; FISCHER, Frida M. Assédio moral no trabalho e suas representações na mídia jornalística. Revista de Saúde Pública, São Paulo, v. 46, n. 3, p. 417-424, 2012.

GARCIA, Átala L. et al. Índices "notícia-morbidade" e notícia-morte": relação entre o perfil de morbimortalidade da população e a divulgação midiática no Estado do Espírito Santo, Brasil, 2011-2012. Revista Brasileira de Pesquisa em Saúde, Vitória, v. 21, n. 1, p. 35-45, 2019.

GARTZIA, Leire; PIZARRO, Jon; BANIANDRES, Josune. Emotional Androgyny: A Preventive Factor of Psychosocial Risks at Work?. Frontiers in psychology, Belgium, v.9, nov. 2018.

HACKNEY, Kaylee J; PERREWÉ, Pamela L. A review of abusive behaviors at work: the development of a process model for studying abuse. Organizacional Psychology Review, New Zealand, v. 8, n. 1, p. 70-92, 2018.

HÅKANSSON, Carita; AHLBORG Jr. Gunnar. Occupational imbalance and the role of perceived stress in predicting stress-related disorders.
Scandinavian Journal of Occupational Therapy, s.l., v. 25, n. 4, p. 278-287, 2018.

HAUSMANN, Ricardo et al. The Atlas of Economic Complexity: Mapping Paths to Prosperity. Cambridge, MA: Mit Press, 2014, p. 91.

INSTITUTO BRASILEIRO DE GEOGRAFIA E ESTATÍSTICA(IBGE). PNAD Contínua: taxa de desocupação é de $12,7 \%$ e taxa de subutilização é de $25,0 \%$ no trimestre encerrado em março de 2019. Disponível em: <https:// agenciadenoticias.ibge.gov.br/agencia-sala-de-imprensa/2013-agencia-de-noticias/ releases/24284-pnad-continua-taxa-de-desocupacao-e-de-12-7-e-taxa-de-subutilizacao-e-de-25-0-no-trimestre-encerrado-em-marco-de-2019. Acesso em: 03/2019.

JAMES, Stephen M. et al. Shift work: disrupted circadian rhythms and sleep-implications for health and well-being. Current Sleep Medicine Reports, Switzerland, v. 3, n. 2, p. 104-112, 2017.

KAKUI, Irene M. Effects of career development on employee performance in the public sector: A case of national cereals and produce board. Strategic Journal of Business \& Change Management, India, v. 3, n. 3, p. 307-324, 2016.

KOMPIER, Michiel A. J.; COOPER Cary L.; GEURTS, Sabine A. E. A multiple case study approach to work stress prevention in Europe. European Journal of Work and Organizational Psychology, Germany, v. 9, n. 3, Volume 9, p. 371-400, 2000.

KRÖLL, Claudia; DOEBLER, Philipp; NÜESCH, Stephan. Meta-analytic evidence of the effectiveness of stress management at work. European Journal of Work and Organizational Psychology, Germany, v. 26, n. 5, p. 677-693, 2017.

KUŞ SAILLARD, Elif. Systematic versus interpretive analysis with two CAQDAS packages: NVivo and MAXQDA. Forum: Qualitative Social Research, s.I., v. 12, n. 1, Jan. 2011.

LANGBECKER, Andrea; CASTELLANOS, Marcelo E. P.; CATALAN-MATAMOROS, Daniel. O que os valores-notícia podem nos dizer sobre o Sistema Único de Saúde? Explorando aportes teórico-conceituais da noticiabilidade. Interface: 
Comunicação, Saúde, Educação, Botucatu, v. 23, 2019.

LEKA, Stavroula; GRIFFITHS, Amanda; COX, Tom. Work organization \& stress: systematic problem approaches for employers, managers and trade union representatives. Reino Unido: World Health Organization, 2004. (Protecting Workers'Health Series, n. 3). Disponível em: <https://www.who. int/occupational_health/publications/pwh3rev. pdf>. Acesso em: 9 set. 2019.

LERNER, Kátia. Doença, mídia e subjetividade: algumas aproximações teóricas. In: LERNER, Kátia; SACRAMENTO, Igor. Saúde e jornalismo: interfaces contemporâneas. Rio de Janeiro: Fiocruz; 2014. p. 151-164.

LEWIS, Justin; WILLIAMS, Andrew; FRANKLIN, Bob. A compromised fourth estate? United Kingdom news journalism, public relations and news sources. Journal Journalism Studies, Routledge, v. 9, n. 1, p. 1-20, 2008.

LUA, Iracema et al. Autoavaliação negativa da saúde em trabalhadoras de enfermagem da atenção básica. Trabalho, Educação e Saúde, Rio de Janeiro, v. 16, n. 3, p. 1.301-1.319, dez. 2018.

LUIZ, Olinda do Carmo. Ciência e risco à saúde nos jornais diários. São Paulo: Annablume; São Bernardo: Cesco, 2006. 182 p.

MACHADO, Izamara B. Percepções sobre o SUS: o que a mídia mostra e o revelado em pesquisa. In: LERNER Kátia, SACRAMENTO Igor. Saúde e jornalismo: interfaces contemporâneas. Rio de Janeiro: Fiocruz, 2014, p. 235-250.

MARCELINO FILHO, Arthur; ARAÚJO, Tania M. Estresse ocupacional e saúde mental dos profissionais do Centro de Especialidades Médicas de Aracaju. Trabalho, Educação e Saúde, Rio de Janeiro, v. 13, p. 177-199, 2015. Suplemento 1.

MARK, George M. The relationship between workplace stress, and job characteristics, individual differences, and mental health. 2008. 316f. Tese (Doutorado em Psicologia) - School of Psychology, Cardiff University, Cardiff, 2008. Disponível em: <http://orca.cf.ac.uk/54730/> Acesso em: 11 set. 2019.
MARTINS, Maria I. C.; MOLINARO, Alex. Reestruturação produtiva e seu impacto nas relações de trabalho nos serviços públicos de saúde no Brasil. Ciência \& Saúde Coletiva, Rio de Janeiro, v. 18, n. 6, p. 1667-1676, 2013.

MEDEIROS, Patrícia F;; GUARESCHI, Neuza M. F. A mídia como ferramenta de pesquisa: produção de saberes no cotidiano sobre a saúde das filhas deste solo. Psicologia \& Sociedade, Porto Alegre, v. 20, p. 87-95, 2008. (Número Especial).

MELO, Cynthia F.; CAVALCANTE, Ana K. S.; FACANHA, Klediane Q. Invisibilização do adoecimento psíquico do trabalhador: limites da integralidade na rede de atenção à saúde. Trabalho, Educação e Saúde, Rio de Janeiro, v. 17, n. 2, 2019.

MENDES, Ana M. B. A organização do trabalho como produto da cultura e a prevenção do estresse ocupacional: $\mathrm{O}$ olhar da psicodinâmica do trabalho. In TAMAYO, Álvaro (Org.). Estresse e cultura organizacional. São Paulo: Casa do Psicólogo, 2008. p. 164-192.

MILCZAREK, Malgorzata; SCHNEIDER, Elke; RIAL GONZÁLEZ, Eusebio. OSH in Figures: stress at work: facts and figures. Luxembourg: Publications Office of the European Union, 2009. (European agency for safety and health at work, 9).

MONTEIRO, Suzana; MARQUES-PINTO, Alexandra. Journalists' occupational stress: a comparative study between reporting critical events and domestic news. The Spanish Journal of Psychology, Cambridge, v. 20, E34, p. 33, 2017.

MORAES FILHO, lel M.; ALMEIDA, Rogério J. Estresse ocupacional no trabalho em enfermagem no Brasil: uma revisão integrativa. Revista Brasileira em Promoção da Saúde. Fortaleza, v. 29, n. 3, p. 447-454, jul./set., 2016.

NIEMANN, Jana et al. When uncertainty counteracts feedback seeking: The effects of interpersonal uncertainty and power on direct feedback seeking. European Journal of Work and Organizational Psychology, Germany, v. 24, n. 2, p. 211-224, 2015.

NOGUEIRA, José H.V; FREITAS, Lêda G. Psicodinâmica do estresse: estudo com trabalhadores de pesquisa, desenvolvimento e inovação. Revista 
Psicologia Organizações e Trabalho, Brasília, v.15, n. 2, p. 133-145, 2015.

OLIVEIRA, Valdir C. A comunicação midiática e o Sistema Único de Saúde. Interface (Botucatu), v. 4, n. 7, p. 71-80, 2000.

OLIVEIRA, Valdir C. As fabulações jornalísticas e a saúde. In: LERNER, Kátia; SACRAMENTO, Igor. Saúde e jornalismo: interfaces contemporâneas. Rio de Janeiro: Fiocruz; 2014. p. 35-60.

OLIVEIRA, Bruno L. C. A. de; SILVA, Alécia M. da; LIMA, Sara F. Carga semanal de trabalho para enfermeiros no Brasil: desafios ao exercício da profissão. Trabalho, Educação e Saúde, Rio de Janeiro, v. 16, n. 3, p. 1.221-1.236, dez. 2018.

ORTONA, Concília; FORTES, Paulo A. C. Jornalistas que escrevem sobre saúde conhecem a humanização do atendimento? Saúde e Sociedade, São Paulo, v. 21, n. 4, p. 909-915, 2012.

PEREIRA, Luciano Z.; ZILLE, Giancarlo P. O estresse no trabalho: uma análise teórica de seus conceitos e suas inter-relações. Gestão e Sociedade, v. 4, n. 7, p. 414-434, 2010.

PROOST, Karin et al. Work-family conflict and facilitation: The combined influence of the job demand-control model and achievement striving. European Journal of Work and Organizational Psychology, Germany, v. 19, n. 5, p. 615-628, 2010.

RAMAZZINI, Bernardino. As doenças dos trabalhadores. 4. ed. São Paulo: Fundacentro, 2016. p. 343.

RICHARDSON, Katherine M. Managing employee stress and wellness in the new millennium. Journal of Occupational Health Psychology, United States, v. 22, n. 3, p. 423-428, 2017.

RODRIGUES, Adriano D. Delimitação, natureza e funções do discurso midiático. In: MOUILLAUD, Maurice; PORTO, Sérgio D. (org.). O jornal, da forma ao sentido. Brasília: Paralelo 15, 1997. p. 218-237.

RODRIGUES, Adriano D. Entrevista: em busca das especificidades do campo jornalístico. Revista Pauta Geral: estudos em jornalismo,
Ponta Grossa, v. 1, n. 1, p. 102-107, jan./jul. 2014 Disponível em: <https://www.revistas2.uepg. br/index.php/pauta/article/view/6079/3723>. Acesso em: 12 jun. 2016.

RODRIGUES, Cláudia C. F. M. et al. Stress among nursing team members. Journal of Nursing UFPE on line. Recife, v. 11, n. 2, p. 601-608, fev. 2017.

ROTTA, Ehideé I. G. L. et al. Análise do discurso da 'segurança' na área da saúde: uma crítica ao trabalhador como vigilante de si. Trabalho Educação e Saúde, Rio de Janeiro, v. 16, n. 3, p. 1.361-1.380, dez. 2018.

SCHYNS, Birgit; VAN VELDHOVEN, Marc. Group leadership climate and individual organizational commitment. A multlilevel analysis. Journal Personality and Social Psychology, Michigan, v. 9, n. 2, p. 57-68, 2010.

SELYE, Hans. A syndrome produced by diverse nocuous agents. Nature, v. 138, n. 32, p.230, 1936

SELYE, Hans The stress of life. New York, NY: McGraw-Hill; 1956.

SETTON, Maria da G. Mídia e educação. 1. ed, 1. reimp. São Paulo; ed. Contexto, 2011. 128 p.

SHOEMAKER, Pamela J.; COHEN, Akiba. News around the world: Practitioners, Content, and the Public. New York: Routledge, 2006. 409p.

SILVA, Marcos P. Seleção noticiosa, critérios de noticiabilidade e valores-notícia. In: FERNANDES, Mario L., SILVA, Gislene; SILVA, Marcos P. (org.). Critérios de noticiabilidade: problemas conceituais e aplicações. Florianópolis: Insular, 2014. p. 95-107.

SILVA, Marconi. A notícia como narrativa e discurso. Estudos em Jornalismo e Mídia, v. 4, n. 1, p. 49-64, 2007.

SILVA, Thalita M. et al. A Saúde e o Sistema Único de Saúde nos bastidores da imprensa: o que os jornalistas têm a nos dizer? Revista Brasileira de Pesquisa em Saúde, v. 20, v. 2, p. 64-73, 2018.

SMOKTUNOWICZ, Ewelina; CIEŚLAK, Roman. How job and family demands impact change in 
perceived stress: A dyadic study. International Journal of Occupational Medicine and Environmental Health, v. 31, n. 2, p. 199-215, 2018.

SPINK, Mary J. P. O estudo empírico das representações sociais. In: SPINK, Mary J. P. (ed.), O conhecimento no cotidiano: as representações sociais na perspectiva da psicologia social. São Paulo: Brasiliense, 1993. p. 311.

SPINK, Mary J. P. Contribuições da psicologia discursiva para o campo da comunicação sobre riscos em saúde. Reciis: Revista Eletrônica de Comunicação, Informação e Inovação em Saúde, Rio de Janeiro, v. 13, n. 1, p. 7-12, jan.-mar. 2019.

STAJKOVIC, Alexandre D; LUTHANS, Fred. Behavioral management and task performance in organizations: conceptual background, meta-analysis, and test of alternative models. Personnel Psychology, New Jersey, v. 56, n. 1, p. 155-194, 2003.

STEYN, Renier; VAWDA, Naseema. Job characteristics: their relationship to job satisfaction, stress and depression. Journal of psychology in Africa (south of the Sahara, the Caribbean, and Afro-Latin America), South Africa, v. 24, n. 3, p. 281-284, 2014.

SUGAWARA, Norio et al. Work-family conflict as a mediator between occupational stress and psychological health among mental health nurses in Japan. Neuropsychiatric disease and treatment, United Kingdom, v. 13, n. 13, p. 779-784, 2017.
THOMPSON, John B. Ideologia e cultura moderna: teoria social crítica na era dos meios de comunicação de massa. Petrópolis: Vozes, 2007. 427p.

TURATO, Egberto R. Métodos qualitativos e quantitativos na área de saúde: definições, diferenças e seus objetos de pesquisa. Revista de Saúde Pública, São Paulo, v. 39, n. 3, p. 507-514, 2005.

TURATO, Egberto R. Tratado da metodologia da pesquisa clínico qualitativa: construção teórico-epistemológica, discussão comparada e aplicação nas áreas da saúde e humanas. 6 . ed. Petrópolis: Vozes; 2011. p. 688.

VAN DIJK, Teun. A. New(s) racism: a discourse analytical approach. In: COTTLE, Simon. Ethnic minorities and the media: changing cultural boundaries. Philadelphia: Open University Press, 2000, p. 33-49.

WANG, Lianwen et al. Media alert in an SIS epidemic model with logistic growth. Journal of Biological Dynamics, Arizona, v. 11; Issue supl; p. 120-137, 2017.

ZAVALIS, Andrea et al. A influência dos fatores estressores sobre os níveis de atenção de profissionais de enfermagem. Revista de Pesquisa: cuidado é fundamental (Online), v. 7, n. 4, p. 3.375-3.387, 2015.

ZEYTINOGLU, Izik U. et al. Health and safety matters! Associations between organizational practices and personal support workers' life and work stress in Ontario, Canada. BMC Health Services Research, California, v. 17, n. 1, p. 427, 2017. 